\title{
Influence of morphology of hollow silica-alumina composite spheres on their activity for hydrolytic dehydrogenation of ammonia borane
}

\author{
Naoki TOYAMA ${ }^{a}$, Ryota OGAWA ${ }^{a}$, Haruki INOUE ${ }^{a}$, Shinobu $\mathrm{OHKI}^{b}$, Masataka \\ TANSHO $^{b}$, Tadashi SHIMIZU ${ }^{b}$, Tetsuo UMEGAKI ${ }^{a,}$, Y Yoshiyuki KOJIMA $^{a}$ \\ ${ }^{a}$ Department of Materials \& Applied Chemistry, College of Science \& Engineering, Nihon University, 1-8-14, \\ Kanda-Surugadai, Chiyoda-Ku, Tokyo 101-8308, Japan \\ ${ }^{b}$ National Institute for Materials Science, 3-13, Sakura, Tsukuba, Ibaraki 305-0003, Japan
}

Received: May 17, 2017; Revised: September 11, 2017; Accepted: September 21, 2017

(C) The Author(s) 2017. This article is published with open access at Springerlink.com

\begin{abstract}
Hollow silica-alumina composite spheres were prepared by a polystyrene (PS) template method using various amounts of PS suspension. Homogeneous hollow spheres prepared using $40 \mathrm{~g}$ were found to be with a diameter of about $300 \mathrm{~nm}$ in scanning electron microscopy, and transmission electron microscopy demonstrated their hollow sphere morphology. From the nitrogen adsorption isotherm results, the homogeneous hollow spheres prepared using $40 \mathrm{~g}$ of the PS suspension were found to be an ordered pore structure. The activities of the hollow spheres prepared using various amounts of the PS suspension for hydrolytic dehydrogenation of ammonia borane were compared. The results showed that 10,7 , and 6 $\mathrm{mL}$ of hydrogen were evolved from the aqueous ammonia borane solution in about $40 \mathrm{~min}$ in the presence of the hollow spheres prepared using 40, 80, and $120 \mathrm{~g}$ of PS suspension, respectively. The homogeneous hollow spheres with an ordered pore structure showed the highest activity among all the hollow spheres. The amount of acid sites and the coordination number of aluminum active species were characterized using neutralization titration and solid-state ${ }^{27} \mathrm{Al}$ magic angle spinning nuclear magnetic resonance spectroscopy. The homogeneous hollow spheres with an ordered pore structure had high amount of acid sites and 4-coordinated aluminum species. The relative proportion of 4-coordinated aluminum species was related to the dispersion of aluminum species. These results indicate that the homogeneous hollow spheres with an ordered pore structure showed the high activity because of high amount of acid sites induced by the highly dispersed aluminum species.

Keywords: ammonia borane; morphology; hollow silica-alumina composite spheres; dispersion; acid sites
\end{abstract}

\section{Introduction}

Hydrogen has been considered for use as a clean energy carrier [1,2]. The search for effective and safe hydrogen storage materials is one of the most difficult challenges for the development of a hydrogen society in the future

*Corresponding author.

E-mail: umegaki.tetsuo@nihon-u.ac.jp
[3-5]. Boron-nitrogen containing compounds are expected to find applications in hydrogen storage materials because of their high hydrogen density $[6,7]$. In particular, ammonia borane $\left(\mathrm{NH}_{3} \mathrm{BH}_{3}\right)$ has attracted a great deal of attention due to its high hydrogen content $(19.6 \mathrm{wt} \%)$, low molecular weight $(30.9 \mathrm{~g} / \mathrm{mol})$, and high stability [8-12]. Additionally, $\mathrm{NH}_{3} \mathrm{BH}_{3}$ releases hydrogen via a hydrolysis reaction in the presence of suitable acids and catalysts at room temperature (Eq. (1)) [13-27]: 


$$
\mathrm{NH}_{3} \mathrm{BH}_{3}+2 \mathrm{H}_{2} \mathrm{O} \rightarrow \mathrm{NH}_{4}^{+}+\mathrm{BO}_{2}^{-}+3 \mathrm{H}_{2}
$$

It has been reported that solid acids such as H-type zeolites (H-BEA and H-MOR) show high activity for the hydrolytic dehydrogenation of $\mathrm{NH}_{3} \mathrm{BH}_{3}$ [13]. We have reported that hollow silica-alumina composite spheres show higher activity compared with fine particles [14].

Hollow spheres have intrinsic features such as easily controllable structure, high specific surface area, low density, and high adsorption capacity. Hollow spheres have been applied in many applications such as catalysis, energy storage, biosensors, and environmental remediation [28-32]. We have expected that the hollow spheres reveal the relationship between the catalytic activity and geometrical structure because their geometrical structure can be controlled such as shell thickness, particle size, and the size of pores in the shell. Hollow spheres have been prepared using many templates such as silica particles, carbon particles, bacteria, and polymer particles [33-37]. In particular, polystyrene (PS) particles have attracted a great deal of attention as templates because they are easily removed using either heat treatment or solvent dissolution, and they produce well-defined and monodispersed hollow spheres [38,39].

In a previous study, the Brønsted acid sites of silica-alumina composites were found to promote the hydrolysis reaction of $\mathrm{NH}_{3} \mathrm{BH}_{3}$. The Brønsted acid sites of silica-alumina composites derive from the substitution of silicon for aluminum in silica network $[40,41]$. We believe that the dispersion of aluminum species plays an important role in their activity for the hydrolytic dehydrogenation of $\mathrm{NH}_{3} \mathrm{BH}_{3}$. In this study, we investigate the influence of the morphology of the hollow spheres on the dispersion of the aluminum species, and their activity for the hydrolytic dehydrogenation of $\mathrm{NH}_{3} \mathrm{BH}_{3}$.

\section{Experiments}

\section{1 Preparation of the hollow spheres}

Hollow silica-alumina composite spheres were prepared using the PS template method, as shown in Fig. 1. The monodisperse PS particles were prepared by emulsifier-free emulsion polymerization as follows: 54 $\mathrm{mL}$ of styrene (Kanto Chem. Co., >99.0\%), $9.00 \mathrm{~g}$ of poly(vinyl pyrrolidone) K30 (Fluka, MW $\approx 40000$ ), $1.56 \mathrm{~g}$ of cationic initiator 2,2 '-azodiisobutyramidine dihydrochloride (Kanto Chem. Co., > 95.0\%), and 600 $\mathrm{mL}$ of ion-exchanged water were added to a $1000 \mathrm{~mL}$ three-neck flask equipped with a mechanical stirrer, a thermometer with a temperature controller, a nitrogen $\left(\mathrm{N}_{2}\right)$ inlet, and a Graham condenser, and the flask was placed in an oil bath. The reaction solution was deoxygenated by bubbling nitrogen gas through the solution at room temperature for $1 \mathrm{~h}$. The solution was stirred at $343 \mathrm{~K}$ for $24 \mathrm{~h}$ at a rate of $250 \mathrm{rpm}$. The obtained PS suspension was centrifuged at $6000 \mathrm{rpm}$ for $5 \mathrm{~min}$ and washed three times with ethanol (Kanto Chem. Co., >99.5\%), and the content of the PS suspension could be tailored through the addition of ethanol. The hollow silica-alumina composite spheres were prepared using a sol-gel method as follows: $0.0228 \mathrm{~g}$ of aluminum isopropoxide (Kanto Chem. Co., $>99.0 \%), 27 \mathrm{~mL}$ of ion exchanged water, $0.4650 \mathrm{~g}$ of $\mathrm{L}(+)$-arginine (Wako Pure Chemical, >98.0\%), $160 \mathrm{~mL}$ of ethanol, and $0.6204 \mathrm{~mL}$ of tetraethoxysilane (TEOS, Kanto Chem. Co., > 99.9\%) were added to 40-120 g of the PS suspension. The sol-gel reaction was carried out at $323 \mathrm{~K}$ for $17 \mathrm{~h}$, and the as-prepared composites were obtained. After drying in a desiccator overnight, the hollow spheres were obtained by calcination in air by raising the temperature to $873 \mathrm{~K}$ at a rate of $0.5 \mathrm{~K} / \mathrm{min}$, followed by cool down immediately after the designated temperature was reached.

\section{2 Characterization}

The morphology of the hollow silica-alumina composite spheres was observed using a scanning electron microscope (SEM, Hitachi S-4500) operating at an acceleration voltage of $15 \mathrm{kV}$ and a transmission electron microscope (TEM, JEOL JEM-2000EX) operating at an acceleration voltage of $200 \mathrm{kV}$. The specific surface area and porosity of the hollow spheres were measured by $\mathrm{N}_{2}$ sorption at $77 \mathrm{~K}$ (BELSORP-max, microtracBEL). The adsorption isotherm and pore size distribution of the hollow spheres were calculated using Barrett-Joyner-Halenda (BJH) and Saito-Foley

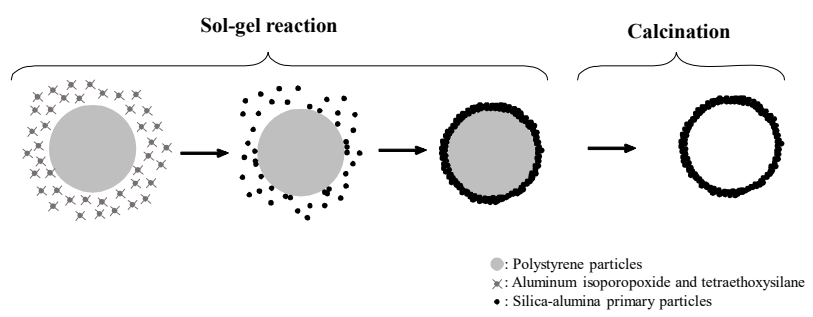

Fig. 1 Schematic illustration of the formation process of the hollow silica-alumina composite spheres. 
(SF) methods. The BJH method calculated the pore size distribution to be in the range of $2-50 \mathrm{~nm}$ based on the Kelvin equation, while the SF method calculated the pore size distribution to be in the range of 0.4-2.0 nm based on the Horvath-Kawazoe method $[42,43]$. The solid-state ${ }^{27} \mathrm{Al}$ magic angle spinning nuclear magnetic resonance (MAS NMR, JEOL ECA-800) spectra were recorded using an 18.8 tesla NMR spectrometer. The relaxation delay time was $2 \mathrm{~s}$, and the hollow spheres were spun at $20 \mathrm{kHz}$ using a $3.2 \mathrm{~mm} \mathrm{ZrO}_{2}$ rotor. The chemical shift was referenced to $1.0 \mathrm{M}$ aqueous aluminum chloride (Wako Pure Chemical, $>98.0 \%$ ) solution. The amount of acid sites for the hollow spheres was measured by neutralization titration with n-butyl amine (Kanto Chem. Co.). The hollow spheres $(0.2 \mathrm{~g})$ were dispersed in $20 \mathrm{~mL}$ of ethanol under sonication. The suspension was titrated with $0.1 \mathrm{M}$ n-butylamine using methyl red (Kanto Chem. Co.) as an indicator. The amount of acid sites of the hollow spheres was calculated as follows: the amount of $0.1 \mathrm{M}$ n-butylamine was divided by the amount of hollow spheres.

\section{3 Activity for the hydrolytic dehydrogenation of $\mathrm{NH}_{3} \mathrm{BH}_{3}$}

The hollow spheres $(0.4 \mathrm{~g})$ were placed in a two-necked round-bottomed flask in air at room temperature; one neck was connected to a gas burette, and the other was connected to an addition funnel. The reaction was started by stirring the mixture of the hollow spheres, and aqueous $\mathrm{NH}_{3} \mathrm{BH}_{3}$ (Aldrich, 90\%) solution $(0.14 \mathrm{wt} \%, 3.5 \mathrm{~mL})$ was added from the addition funnel. The evolution of gas was monitored using the gas burette, as shown in Fig. 2.

\section{Results and discussion}

The morphology of the hollow spheres was observed using SEM and TEM measurements. Figure 3 shows SEM and TEM images of the composites prepared using various amounts of PS suspension. All the spherical particles were observed to be a diameter of about $300 \mathrm{~nm}$ in the SEM images, and the inset TEM image demonstrates their hollow morphology (Fig. 3(a)). When $40 \mathrm{~g}$ of PS suspension, homogeneous hollow spheres were obtained. In contrast, some of the hollow spheres prepared using 80 and $120 \mathrm{~g}$ of PS suspension were collapsed, as shown in Figs. 3(b) and

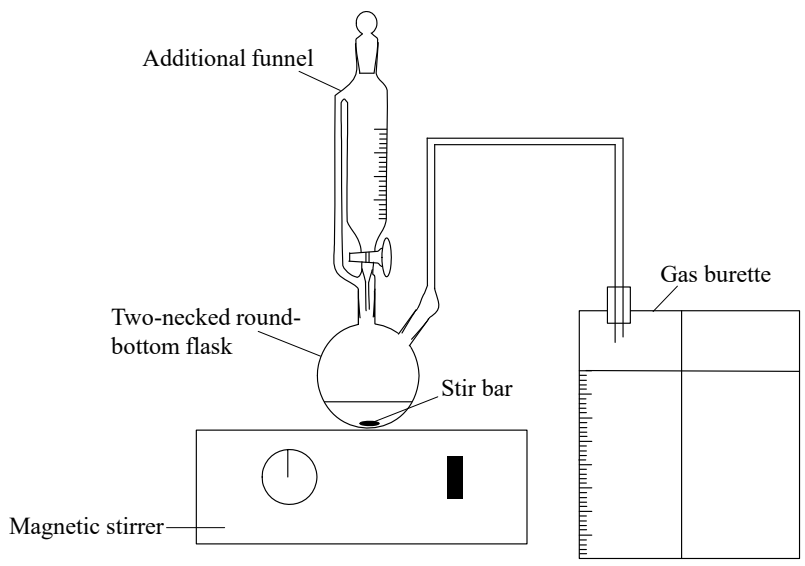

Fig. 2 Schematic illustration of the hydrolytic dehydrogenation of $\mathrm{NH}_{3} \mathrm{BH}_{3}$.
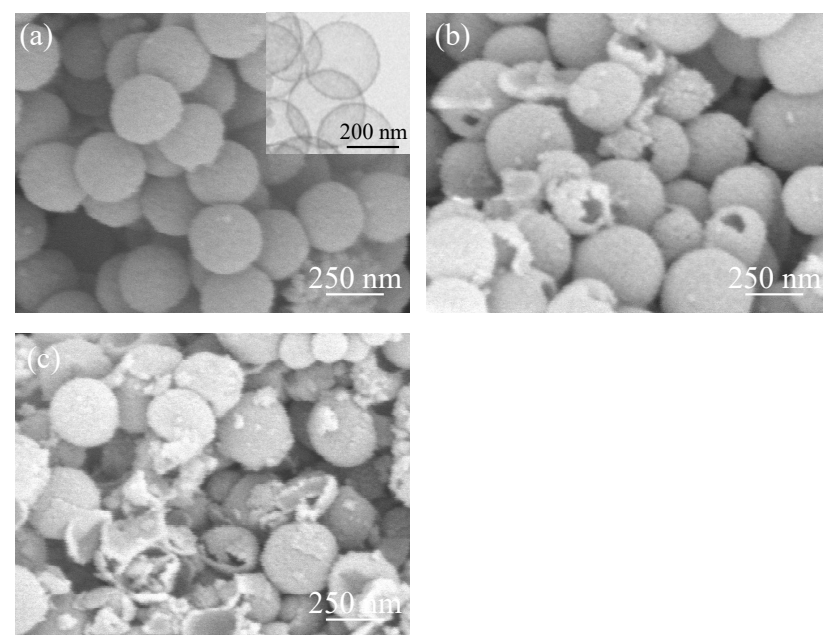

Fig. 3 SEM and TEM images of the silica-alumina composites prepared using (a) 40, (b) 80, and (c) $120 \mathrm{~g}$ of the PS suspension.

3(c). The hollow spheres were prepared using a PS template method as described previous study [39,44-46]. In this method, silica-alumina composite primary particles were prepared using a sol-gel method, and the primary particles were deposited on the surface of PS template particles to form composite shells $[44,47-49]$. The result suggests that the primary particles determine the pore size and pore size distribution in the shells of the hollow spheres, as shown in Fig. 1.

The porosity of the hollow spheres prepared using various amounts of the PS suspension were determined using $\mathrm{N}_{2}$ sorption. Figure 4 shows the $\mathrm{N}_{2}$ adsorption-desorption isotherms of the hollow spheres prepared using various amounts of the PS suspension. All the hollow spheres showed hysteresis loops in the relative pressure range of $0.5-1.0$. The isotherms of all 


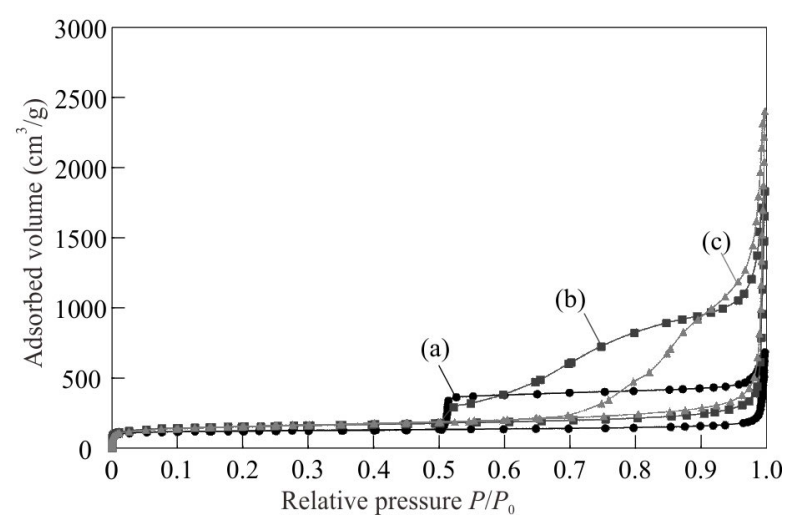

Fig. $4 \quad \mathrm{~N}_{2}$ sorption isotherms of the hollow silica-alumina composite spheres prepared using (a) 40, (b) 80 , and (c) $120 \mathrm{~g}$ of the PS suspension.

the hollow spheres were classified as being type IV isotherms according to the IUPAC classification system for physisorption isotherms, suggesting that the hollow spheres had mesoporous shell. Many different shapes of hysteresis loops have been reported, and the type of hysteresis loop observed is closely related to the particular features of the pore structure and the underlying adsorption mechanism [50,51]. The hollow spheres prepared using $40 \mathrm{~g}$ of PS suspension demonstrated a type H1 loop, which is associated with an ordered pore structure. On the other hand, the hollow spheres prepared using 80 and $120 \mathrm{~g}$ of PS suspension exhibited type $\mathrm{H} 2$ loops, which are associated with a disordered pore structure. The pore size distribution was calculated by using the adsorption branch data of the isotherm. Figure 5 shows the pore size distribution calculated using the BJH method for the hollow spheres prepared using various amounts of the PS suspension. The pore size distributions of the hollow spheres prepared using 40, 80, and $120 \mathrm{~g}$ of PS

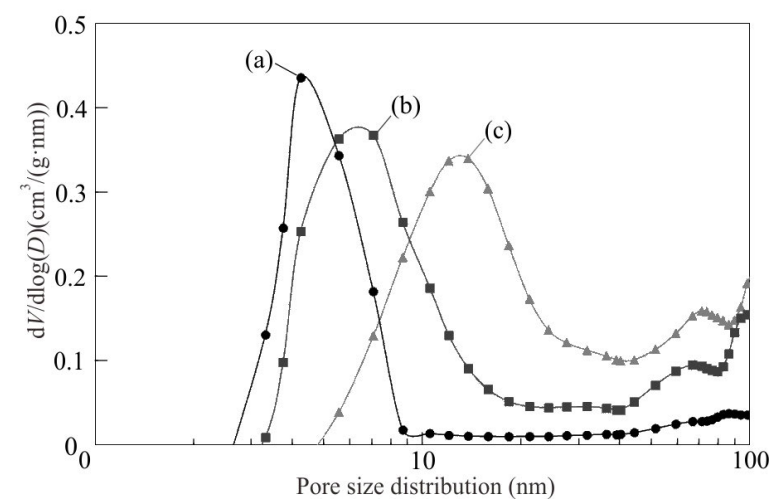

Fig. 5 Pore size distribution of the hollow silica-alumina composite spheres prepared using (a) 40, (b) 80 , and (c) $120 \mathrm{~g}$ of the PS suspension, calculated using the BJH method. suspension were centered at around 4.2, 7.1, and $13.8 \mathrm{~nm}$, respectively. Based on this result, the pore size of the hollow spheres increased with increasing amounts of the PS suspension. This was attributed to an increase in the interparticle distance between the primary particles in the shell of the hollow spheres with increasing the amounts of the PS suspension. Figure 6 shows the pore size distribution calculated using the SF method for the hollow spheres prepared using various amounts of the PS suspension. Based on this method, the pore size distributions of all the hollow spheres were centered at $0.43 \mathrm{~nm}$. The result indicated that the primary particles probably showed similar pore size distributions.

Activities of the hollow spheres prepared using various amounts of the PS suspension for the hydrolytic dehydrogenation of $\mathrm{NH}_{3} \mathrm{BH}_{3}$ were compared. Figure 7 shows the time course of hydrogen evolution in the presence of the hollow spheres

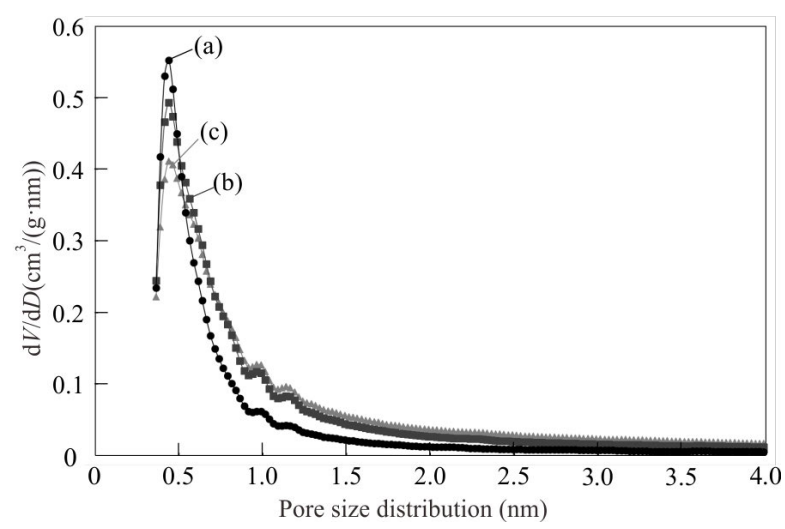

Fig. 6 Pore size distribution of the hollow silica-alumina composite spheres prepared using (a) 40, (b) 80, and (c) $120 \mathrm{~g}$ of the PS suspension, calculated using the SF method.

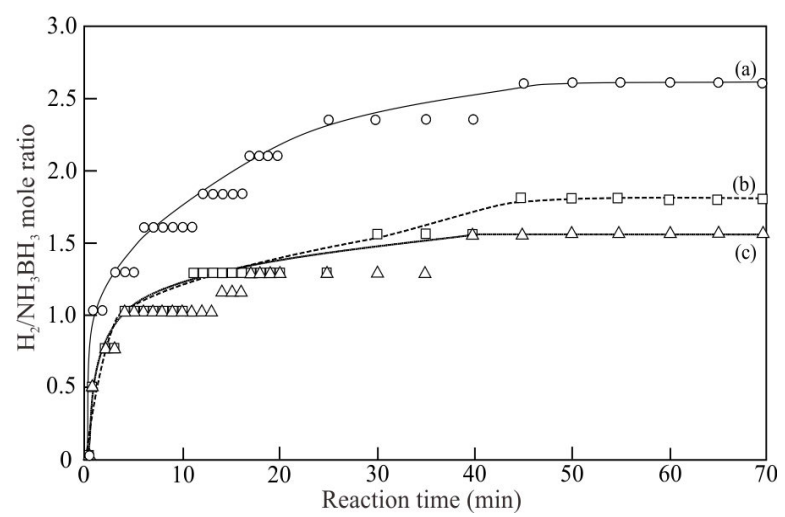

Fig. $7 \quad \mathrm{H}_{2} / \mathrm{NH}_{3} \mathrm{BH}_{3}$ molar ratios of hydrogen generated from an aqueous $\mathrm{NH}_{3} \mathrm{BH}_{3}$ solution $(0.14 \mathrm{wt} \%, 3.5 \mathrm{~mL})$ in the presence of the hollow silica-alumina composite spheres prepared using (a) 40, (b) 80 , and (c) $120 \mathrm{~g}$ of the PS suspension. 
prepared using various amounts of the PS suspension. As shown, the hydrogen evolution of 10,7 , and $6 \mathrm{~mL}$ was completed in 45,45 , and $40 \mathrm{~min}$ in the presence of the hollow spheres prepared using 40,80 , and $120 \mathrm{~g}$ of the PS suspension, respectively. The molar ratios of the hydrolytically evolved hydrogen to the initial $\mathrm{NH}_{3} \mathrm{BH}_{3}$ in the presence of the hollow spheres prepared using 40, 80, and $120 \mathrm{~g}$ of the PS suspension were $2.6,1.8$, and 1.6, respectively. As the theoretical $\mathrm{H}_{2} / \mathrm{NH}_{3} \mathrm{BH}_{3}$ molar ratio was 3.0, the reaction is almost completed in the presence of the homogeneous hollow spheres prepared using $40 \mathrm{~g}$ of the PS suspension. Hollow silica-zirconia composite spheres [17], hollow silica-alumina composite spheres prepared using aluminum-sec-butoxide [18], and H-BEA zeolites [13] were evaluated for the hydrolytic dehydrogenation of $\mathrm{NH}_{3} \mathrm{BH}_{3}$ as previously reported. The volumes of hydrogen evolved per gram of the hollow spheres were measured for the homogenous hollow spheres prepared using $40 \mathrm{~g}$ of the PS suspension, the hollow silica-zirconia composite spheres, the hollow silica-alumina composite spheres prepared using aluminum-sec-butoxide, and the H-BEA zeolites, and the evolved hydrogen volumes were found to be 6.5 , $2.7, \quad 3.8$, and $4.7 \mathrm{~mL} / \mathrm{g}$, respectively. Thus, the homogenous hollow spheres prepared using $40 \mathrm{~g}$ of the PS suspension showed high activity when compared with previously reported solid acids. The recyclability of the homogenous hollow spheres prepared using $40 \mathrm{~g}$ of the PS suspension in the activity for the hydrolytic dehydrogenation of $\mathrm{NH}_{3} \mathrm{BH}_{3}$ was tested. The recycled hollow spheres almost demonstrated no activity. A possible cause for this deactivation might be the adsorption of ammonium ions on the acid sites of the hollow spheres during the hydrolytic dehydrogenation of $\mathrm{NH}_{3} \mathrm{BH}_{3}$. Additionally, the rate of hydrogen evolution was calculated using the hydrogen evolution data from the start of the reaction until $50 \%$ of the final hydrogen volume had been evolved. The rates of hydrogen evolution of the hollow spheres prepared using 40, 80, and $120 \mathrm{~g}$ of the PS suspension were 0.9, 1.0 , and $1.0 \mathrm{~mL} / \mathrm{min}$, respectively. Thus, the rate of hydrogen evolution was very similar for all the hollow spheres. This result is in agreement with the pore size distribution calculated using the SF method, as shown in Fig. 6.

The coordination numbers of the hollow spheres prepared using various amount of PS suspension were measured using solid-state ${ }^{27} \mathrm{Al}$ MAS NMR, as shown in Fig. 8. Three peaks were observed at around 62, 35,

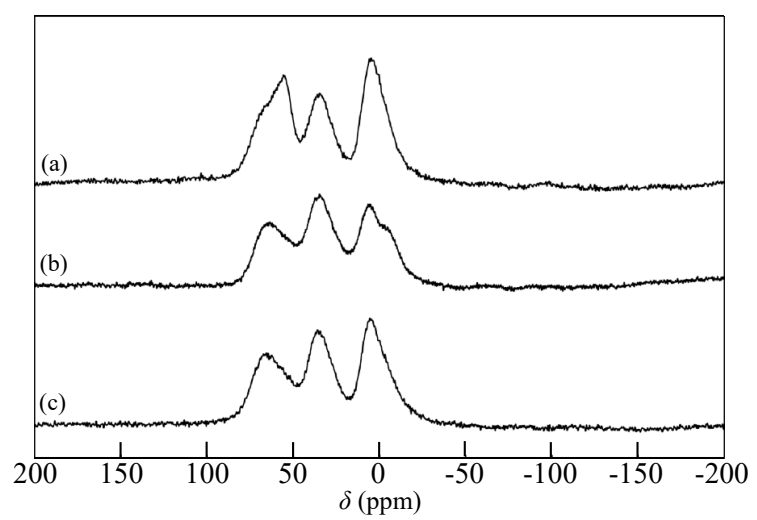

Fig. 8 Solid-state ${ }^{27} \mathrm{Al}$ MAS NMR spectra of the hollow silica-alumina composite spheres prepared using (a) 40, (b) 80 , and (c) $120 \mathrm{~g}$ of the PS suspension.

and $5 \mathrm{ppm}$ in the spectra of all the hollow spheres, corresponding to 4-, 5-, and 6-coordinated aluminum species, respectively [52,53]. The 4-coordinated aluminum species were assigned to aluminum species in the silica matrix, while the 5- and 6-coordinated aluminum species were assigned to aluminum species outside the silica matrix $[54,55]$. It has been reported that 4-coordinated aluminum species were ascribed to Brønsted acid sites, while 5- and 6-coordinated aluminum species were ascribed to Lewis acid sites $[52,55]$. The ratios of the peak area of the 4-coordinated aluminum species to the sum of the peak areas of the 4-, 5-, and 6-coordinated aluminum species $\left(I_{4} / I_{\text {all }}\right)$ in the spectra of the hollow spheres prepared using 40,80 , and $120 \mathrm{~g}$ of the PS suspension were found to be $0.32,0.26$, and 0.24 , respectively. The $I_{4} / I_{\text {all }}$ ratio of the homogeneous hollow spheres prepared using $40 \mathrm{~g}$ of the PS suspension was the highest among all the hollow spheres. It has been reported that 4-coordinated aluminum species are related to the dispersion of aluminum species [53]. These results indicated that the homogeneous hollow spheres prepared using $40 \mathrm{~g}$ of the PS suspension exhibited high dispersion of the aluminum species in the silica matrix.

The amounts of acid sites of the hollow spheres prepared using various amounts of the PS suspension were measured by neutralization titration with n-butyl amine using methyl red as an indicator. The amounts of acid sites of the hollow spheres prepared using 40, 80 , and $120 \mathrm{~g}$ of PS suspension were found to be 1.50 , 1.18 , and $0.91 \mathrm{mmol} / \mathrm{g}$, respectively. The amount of acid sites of the homogeneous hollow spheres prepared using $40 \mathrm{~g}$ of the PS suspension was the highest among all the hollow spheres. This result was attributed to the 
amount of acid sites decreasing as the amount of collapsed hollow spheres increased, due to aggregation between some of the collapsed hollow spheres during the calcination process.

Table 1 shows the physicochemical properties of the hollow spheres prepared using various amounts of the PS suspension. The specific surface area of the hollow spheres increased as the amount of the PS suspension used was increased. The surface concentrations of acid sites in the hollow spheres prepared using 40,80 , and $120 \mathrm{~g}$ of the PS suspension were found to be 3.2, 2.1, and $1.6 \mu \mathrm{mol} / \mathrm{m}^{2}$, respectively. This result suggested that the aluminum species of the homogeneous hollow spheres prepared using $40 \mathrm{~g}$ of PS suspension were highly dispersed in the silica matrix. This result refracted the dispersion of aluminum species, as shown in Fig. 8. The morphology of the hollow spheres played an important role in the dispersion of the aluminum species. The results indicated that the homogeneous hollow spheres had large amount of acid sites because of the highly dispersed aluminum species.

\section{Conclusions}

In this study, we investigated the influence of the morphology of hollow silica-alumina composite spheres on their activity for the hydrolytic dehydrogenation of $\mathrm{NH}_{3} \mathrm{BH}_{3}$. The morphology of the hollow spheres was influenced by the amount of the PS suspension used in their preparation. Homogeneous hollow spheres were obtained when $40 \mathrm{~g}$ of the PS suspension was used to prepare the hollow spheres, as shown in their SEM images. The amount of collapsed hollow spheres increased as the amount of the PS suspension used increased. From the $\mathrm{N}_{2}$ adsorption isotherms, the hollow spheres prepared using $40 \mathrm{~g}$ of the PS suspension were found to be an ordered pore structure, while hollow spheres with a disordered pore structure were obtained when 80 and $120 \mathrm{~g}$ of the PS suspension were used. The activities of the hollow

Table 1 Physicochemical properties of the hollow silica-alumina composite spheres prepared using amounts of the PS suspension

\begin{tabular}{cccc}
\hline $\begin{array}{c}\text { PS } \\
\begin{array}{c}\text { suspension } \\
(\mathrm{g})\end{array}\end{array}$ & $\begin{array}{c}\text { Specific surface } \\
\text { area }^{*} S_{\mathrm{BET}}\left(\mathrm{m}^{2} / \mathrm{g}\right)\end{array}$ & $\begin{array}{c}\text { Acid } \\
\text { sites } \\
(\mathrm{mmol} / \mathrm{g})\end{array}$ & $\begin{array}{c}\text { Acid } \\
\text { sites } / S_{\mathrm{BET}} \\
\left(\mu \mathrm{mol} / \mathrm{m}^{2}\right)\end{array}$ \\
\hline 40 & 468 & 1.50 & 3.2 \\
80 & 564 & 1.18 & 2.1 \\
120 & 553 & 0.91 & 1.6 \\
\hline Specific surface area was measured by $\mathrm{N}_{2}$ adsorption using BET method.
\end{tabular}

spheres prepared using various amounts of PS suspension for the hydrolytic dehydrogenation of $\mathrm{NH}_{3} \mathrm{BH}_{3}$ were compared. The homogeneous hollow spheres prepared using $40 \mathrm{~g}$ of PS suspension demonstrated the highest activity among all the hollow spheres. The solid-state ${ }^{27} \mathrm{Al}$ MAS NMR spectra indicated that hydrogen evolution volume increased as the ratio of 4-coordinated aluminum species increased. The 4-coordinated aluminum species were related to dispersion of aluminum species. The amount of hydrogen evolution increased as the amount of acid sites increased from the neutralization titration measurement. These results indicated that the homogeneous hollow spheres with ordered pore structure showed high activity because of the high amount of acid sites induced by the highly dispersed aluminum species.

\section{Acknowledgements}

This work was supported by NIMS and University of Tokyo microstructural characterization platform as a program of "Nanotechnology Platform" of the Ministry of Education, Culture, Sports, Science and Technology (MEXT), Japan. We are grateful to Mr. Deguchi and Ms. Wada for using the solid-state NMR measurement, and Mr. Ito for using the TEM measurement. Additionally, we also thank MicrotracBEL Corp. for using the $\mathrm{N}_{2}$ sorption measurement.

\section{References}

[1] Rand DAJ, Dell RM. The hydrogen economy: A threat or an opportunity for lead-acid batteries? J Power Sources 2005, 144: 568-578.

[2] Rakap M. Poly( $N$-vinyl-2-pyrrolidone)-stabilized palladium-platinum nanoparticles-catalyzed hydrolysis of ammonia borane for hydrogen generation. $J$ Power Sources 2015, 276: 320-327.

[3] Keaton RJ, Blacquiere JM, Baker RT. Base metal catalyzed dehydrogenation of ammonia-borane for chemical hydrogen storage. $J$ Am Chem Soc 2007, 129: 1844-1845.

[4] Huang Z, Autrey T. Boron-nitrogen-hydrogen (BNH) compounds: Recent developments in hydrogen storage, applications in hydrogenation and catalysis, and new syntheses. Energy Environ Sci 2012, 5: 9257-9268.

[5] Li J, Zhu Q-L, Xu Q. Non-noble bimetallic CuCo nanoparticles encapsulated in the pores of metal-organic frameworks: Synergetic catalysis in the hydrolysis of ammonia borane for hydrogen generation. Catal Sci Technol 2015, 5: 525-530.

[6] Luo W, Zakharov LN, Liu S-Y. 1,2-BN cyclohexane: Synthesis, structure, dynamics, and reactivity. J Am Chem 
Soc 2011, 113: 13006-13009.

[7] Cao N, Luo W, Cheng G. One-step synthesis of graphene supported $\mathrm{Ru}$ nanoparticles as efficient catalysts for hydrolytic dehydrogenation of ammonia borane. Int $J$ Hydrogen Energ 2013, 38: 11964-11972.

[8] Hamilton CW, Baker RT, Staubitzc A, et al. B-N compounds for chemical hydrogen storage. Chem Soc Rev 2009, 38: 279-293.

[9] Xu Q, Chandra M. A portable hydrogen generation system: Catalytic hydrolysis of ammonia-borane. $J$ Alloys Compd 2007, 446-447: 729-732.

[10] Lu Z-H, Li J, Zhu A, et al. Catalytic hydrolysis of ammonia borane via magnetically recyclable copper iron nanoparticles for chemical hydrogen storage. Int $J$ Hydrogen Energ 2013, 38: 5330-5337.

[11] Jiang H-L, Xu Q. Catalytic hydrolysis of ammonia borane for chemical hydrogen storage. Catal Today 2011, 170: $56-63$.

[12] Hu L, Zheng B, Lai Z, et al. Room temperature hydrogen generation from hydrolysis of ammonia-borane over an efficient NiAgPd/C catalyst. Int J Hydrogen Energ 2014, 39: 20031-20037.

[13] Chandra M, Xu Q. Dissociation and hydrolysis of ammonia-borane with solid acids and carbon dioxide: An efficient hydrogen generation system. $J$ Power Sources 2006, 159: 855-860.

[14] Toyama N, Ohki S, Tansho $\mathrm{M}$, et al. Influence of morphology of silica-alumina composites on their activity for hydrolytic dehydrogenation of ammonia borane. Journal of the Japan Institute of Energy 2016, 95: 480-486.

[15] Toyama N, Umegaki T, Kojima Y. Fabrication of hollow silica-alumina composite spheres and their activity for hydrolytic dehydrogenation of ammonia borane. Int $J$ Hydrogen Energ 2014, 39: 10136-10143.

[16] Toyama N, Ohki S, Tansho M, et al. Influence of alcohol solvents on morphology of hollow silica-alumina composite spheres and their activity for hydrolytic dehydrogenation of ammonia borane. J Sol-Gel Sci Technol 2017, 82: 92-100.

[17] Umegaki T, Hosoya T, Toyama N, et al. Fabrication of hollow silica-zirconia composite spheres and their activity for hydrolytic dehydrogenation of ammonia borane. $J$ Alloys Compd 2014, 608: 261-265

[18] Toyama N, Ohki S, Tansho $\mathrm{M}$, et al. Influence of aluminum precursors on structure and acidic properties of hollow silica-alumina composite spheres, and their activity for hydrolytic dehydrogenation of ammonia borane. Int J Hydrogen Energ 2017, 42: 22318-22324.

[19] Tong DG, Zeng XL, Chu W, et al. Magnetically recyclable hollow Co-B nanospindles as catalysts for hydrogen generation from ammonia borane. J Mater Sci 2010, 45: 2862-2867.

[20] Fan Y, Li X, He X, et al. Effective hydrolysis of ammonia borane catalyzed by ruthenium nanoparticles immobilized on graphic carbon nitride. Int J Hydrogen Energ 2014, 39: 19982-19989.

[21] Chen W, Ji J, Feng X, et al. Mechanistic insight into size-dependent activity and durability in Pt/CNT catalyzed hydrolytic dehydrogenation of ammonia borane. $J \mathrm{Am}$ Chem Soc 2014, 136: 16736-16739.

[22] Umegaki T, Yan J-M, Zhang X-B, et al. Hollow $\mathrm{Ni-} \mathrm{SiO}_{2}$ nanosphere-catalyzed hydrolytic dehydrogenation of ammonia borane for chemical hydrogen storage. $J$ Power Sources 2009, 191: 209-216.

[23] Cao N, Hu K, Luo W, et al. RuCu nanoparticles supported on graphene: A highly efficient catalyst for hydrolysis of ammonia borane. J Alloys Compd 2014, 590: 241-246.

[24] Yang L, Su J, Meng X, et al. In situ synthesis of graphene supported Ag@CoNi core-shell nanoparticles as highly efficient catalysts for hydrogen generation from hydrolysis of ammonia borane and methylamine borane. $J$ Mater Chem A 2013, 1: 10016-10023.

[25] Yang L, Luo W, Cheng G. Graphene-supported Ag-based core-shell nanoparticles for hydrogen generation in hydrolysis of ammonia borane and methylamine borane. ACS Appl Mater Interfaces 2013, 5: 8231-8240.

[26] Umegaki T, Xu Q, Kojima Y. Effect of L-arginine on the catalytic activity and stability of nickel nanoparticles for hydrolytic dehydrogenation of ammonia borane. J Power Sources 2012, 216: 363-367.

[27] Umegaki T, Takei C, Xu Q, et al. Fabrication of hollow metal oxide-nickel composite spheres and their catalytic activity for hydrolytic dehydrogenation of ammonia borane. Int J Hydrogen Energ 2013, 38: 1397-1404.

[28] Wang C-A, Li S, An L. Hierarchically porous $\mathrm{Co}_{3} \mathrm{O}_{4}$ hollow spheres with tunable pore structure and enhanced catalytic activity. Chem Commun 2013, 49: 7427-7429.

[29] Lin Y-S, Wu S-H, Tseng C-T, et al. Synthesis of hollow silica nanospheres with a microemulsion as the template. Chem Commun 2009, 24: 3542-3544.

[30] Liu J, Li W, Manthiram A. Dense core-shell structured $\mathrm{SnO}_{2} / \mathrm{C}$ composites as high performance anodes for lithium ion batteries. Chem Commun 2010, 46: 1437-1439.

[31] Li M, Xu W, Wang W, et al. Facile synthesis of specific $\mathrm{FeMnO}_{3}$ hollow sphere/graphene composites and their superior electrochemical energy storage performances for supercapacitor. J. Power Sources 2014, 248: 465-473.

[32] Kang N, Park JH, Jin M, et al. Microporous organic network hollow spheres: Useful templates for nanoparticulate $\mathrm{Co}_{3} \mathrm{O}_{4}$ hollow oxidation catalysts. $J \mathrm{Am}$ Chem Soc 2013, 135: 19115-19118.

[33] Dhas NA, Suslick KS. Sonochemical preparation of hollow nanospheres and hollow nanocrystals. $J$ Am Chem Soc 2005, 127: 2368-2369.

[34] Yang S, Zhang B, Ge C, et al. Close-packed mesoporous carbon polyhedrons derived from colloidal carbon microspheres for electrochemical energy storage applications. RSC Adv 2012, 2: 10310-10315.

[35] Chen S, Huang P, Wang Z, et al. Self-assembly of gold nanoparticles to silver microspheres as highly efficient 3D SERS substrates. Nanoscale Res Lett 2013, 8: 168.

[36] Wan H, Long Y, Xu H, et al. New strategy to prepare hollow silica microspheres with tunable holes on the shell Wall. Langmuir 2014, 30: 683-686. 
[37] Nandiyanto ABD, Suhendi A, Ogi T, et al. Synthesis of additive-free cationic polystyrene particles with controllable size for hollow template applications. Colloid Surface A 2012, 396: 96-105.

[38] Shen B-H, Hsieh M-L, Chen H-Y, et al. The preparation of hollow silica spheres with mesoporous shell via polystyrene emulsion latex template and the investigation of ascorbic acid release behavior. $J$ Polym Res 2013, 20: 220.

[39] Nandiyanto ABD, Suhendi A, Ogi T, et al. Size- and charge-controllable polystyrene spheres for templates in the preparation of porous silica particles with tunable internal hole configurations. Chem Eng $J$ 2014, 256: 421-430.

[40] Hensen EJM, Poduval DG, Magusin PCMM, et al. Formation of acid sites in amorphous silica-alumina. $J$ Catal 2010, 269: 201-218.

[41] Gil A, Vicente MA, Korili SA. Effect of the $\mathrm{Si} / \mathrm{Al}$ ratio on the structure and surface properties of silica-alumina-pillared clays. J Catal 2005, 229: 119-126.

[42] Storck S, Bretinger H, Maier WF. Characterization of micro- and mesoporous solids by physisorption methods and pore-size analysis. Appl Catal A 1998, 174: 137-146.

[43] Haghighatju F, Rafsanjani HH. Investigation of activation time on pore size distribution of activated carbon determined with different method. Trans Phenom Nano Micro Scales 2014, 2: 43-47.

[44] Nandiyanto ABD, Akane Y, Ogi T, et al. Mesopore-free hollow silica particles with controllable diameter and shell thickness via additive-free synthesis. Langmuir 2012, 28: 8616-8624.

[45] Ge C, Zhang D, Wang A, et al. Synthesis of porous hollow silica spheres using polystyrene-methyl acrylic acid latex template at different temperatures. $J$ Phys Chem Solids 2009, 70: 1432-1437.

[46] Bao Y, Yang Y, Shi C, et al. Fabrication of hollow silica spheres and their application in polyacrylate film forming agent. J Mater Sci 2014, 49: 8215-8225.

[47] Goodwin JW, Ottewill RH, Pelton R. Studies on the preparation and characterization of monodisperse polystyrene latices V.: The preparation of cationic latices. Colloid \& Polymer Sci 1979, 257: 61-69.
[48] Qiu L, Qu B. Preparation and characterization of surfactant-free polystyrene/layered double hydroxide exfoliated nanocomposite via soap-free emulsion polymerization. $J$ Colloid Interface Sci 2006, 301: 347-351.

[49] Nazaran P, Tauer K. Nucleation in emulsion polymerization: Another step towards non-micellar nucleation theory. Macromol Symp 2007, 259: 264-273.

[50] Thommes M, Kaneko K, Neimark AV, et al. Physisorption of gases, with special reference to the evaluation of surface area and pore size distribution (IUPAC Technical Report). Pure Appl Chem 2015, 87: 1051-1069.

[51] Sing KSW. Reporting physisorption data for gas/solid systems with special reference to the determination of surface area and porosity (Recommendations 1984). Pure Appl Chem 1985, 57: 603-619.

[52] Fang X, Liu Z, Hsieh M-F, et al. Hollow mesoporous aluminosilica spheres with perpendicular pore channels as catalytic nanoreactors. ACS Nano 2012, 6: 4434-4444.

[53] Koekkoek AJJ, Rob van Veen JA, Gerrtisen PB, et al. Brønsted acidity of Al/SBA-15. Microporous Mesoporous Mater 2012, 151: 34-43.

[54] O'Della LA, Savinb SLP, Chadwick AV, et al. A ${ }^{27} \mathrm{Al}$ MAS NMR study of a sol-gel produced alumina: Identification of the NMR parameters of the $\theta-\mathrm{Al}_{2} \mathrm{O}_{3}$ transition alumina phase. Solid State Nucl Magn Res 2007, 31: $169-173$.

[55] Sakthivel A, Dapurkar SE, Gupta NM, et al. The influence of aluminium sources on the acidic behavior as well as on the catalytic activity of mesoporous H-AlMCM-41 molecular sieves. Microporous Mesoporous Mater 2003, 65: 177-187.

Open Access The articles published in this journal are distributed under the terms of the Creative Commons Attribution 4.0 International License (http://creativecommons. org/licenses/by/4.0/), which permits unrestricted use, distribution, and reproduction in any medium, provided you give appropriate credit to the original author(s) and the source, provide a link to the Creative Commons license, and indicate if changes were made. 